\title{
Dose reduction is good but it is image quality that matters
}

\author{
R. Glenn Wells, PhD \\ a University of Ottawa Heart Institute, Cardiac Imaging Program, Ottawa, ON, Canada
}

Received Jul 12, 2018; accepted Jul 12, 2018

doi:10.1007/s12350-018-1378-5

\section{See related article, pp. 228-237}

Dose reduction in nuclear medicine is an important goal. Increased use of imaging, particularly cardiac CT and nuclear medicine has led to a $6 \times$ increase in the population exposure from man-made sources. ${ }^{1}$ This has raised public awareness and increased patient concern over radiation exposure during testing. Although individually, the risk to a patient from any one test is very small, cumulative dose from multiple procedures can become relatively high. In keeping with the principle of ALARA, it is important for us to consider where it is reasonable to reduce dose. ${ }^{2}$

In support of reducing patient exposure in cardiac nuclear medicine, the American Society of Nuclear Cardiology (ASNC) had recommended a goal of having $50 \%$ of myocardial perfusion imaging (MPI) studies performed with $\leq 9 \mathrm{mSv}$ by 2014 . There has been some success in reducing the effective dose, but there remains room for improvement; as of 2013, the median dose in North American was estimated as $12.1 \mathrm{mSv} .^{3}$ Recently released SPECT guidelines ${ }^{4}$ suggest numerous approaches to reducing dose from SPECT MPI including minimizing the use of Tl-201, adjusting the injected activity based on patient weight, using stress-first protocols where appropriate, and employing advanced reconstruction algorithms and new cardiac camera technologies where they are available. Used individually, stress-first protocols, advanced software, and novel hardware solutions can each reduce patient radiation dose from a Tc99m-based study to $\leq 5 \mathrm{mSv} .^{2,3}$ Used in

Reprint requests: R. Glenn Wells, PhD, University of Ottawa Heart Institute, Cardiac Imaging Program, 40 Ruskin St, Ottawa, ON, K1Y4W7 Canada; gwells@ottawaheart.ca

J Nucl Cardiol 2020;27:238-40.

$1071-3581 / \$ 34.00$

Copyright (C) 2018 American Society of Nuclear Cardiology. combination, doses as low as $2.6 \mathrm{mSv}^{5}$ or even less than $1 \mathrm{mSv}$ can be achieved. ${ }^{6}$

However, it is essential that we not let a desire for dose reduction lead us to sacrifice image quality. The risks associated with a poor-quality study, or a patient choosing an inferior test or even opting not to have the test at all due to concern over radiation exposure far outweigh the risks of the radiation exposure itself. ${ }^{7}$ The SPECT guidelines also recommend approaches to maximize the accuracy of the test by addressing common imaging artifacts like attenuation effects. The impact of attenuation artifacts can be reduced by mechanisms like ECG-gated acquisitions and obtaining images in two different patient configurations. These approaches provide additional information without any additional radiation exposure to the patient, but they approach the problem indirectly and may not provide a clear solution. For example, if a suspected attenuation artifact seen on supine imaging partially resolves with prone imaging, does that mean that the remaining reduction in uptake is real or simply that there is attenuation with both patient positions? Attenuation correction (AC) via a transmission map, when done correctly, directly addresses the problem and can accurately resolve the artifact. However, AC requires a transmission map and this is typically a CT scan, which itself adds radiation exposure to the patient.

A regular, prospective ECG-gated CT of the heart for AC delivers $0.3-0.7 \mathrm{mSv}^{8}$ By itself, this does not seem like much radiation compared with the standard $10 \mathrm{mSv}$ associated with a full-dose Tc-99m rest/stress MPI study. However, on a half-dose (185 MBq or $5 \mathrm{mCi}$ ) stress-only protocol, the radiation dose to the patient is $1.3 \mathrm{mSv}$ and adding a $0.7 \mathrm{mSv}$ CT scan increases the patient effective dose by $54 \%$. It is reasonable, therefore, to consider means by which the CT-component of the dose can be reduced in SPECT/CT MPI. There are many parameters that can be adjusted to reduce dose. For example, increasing pitch reduces dose by decreasing the time needed to cover a given axial field-of-view and 
thus decreases the duration of radiation exposure. Increased pitch also spreads out the distance between adjacent acquired slices and may lead to decreased resolution. If the $\mathrm{CT}$ is to be used for other purposes such as localization or calcium scoring, the loss in resolution may not be acceptable. Reducing tube current decreases the number of $\mathrm{x}$-rays produced and thus increases image noise but can reduce patient dose directly in proportion to the reduction in current. Reducing tube voltage, the maximum energy of the $\mathrm{x}$ rays produced, also decreases patient dose, but does so in a non-linear manner. Voltage reductions increase image noise but small changes in voltage have much larger changes in radiation dose.

How much we can afford to increase the noise in the CT scan depends on its effect on image quality. In evaluating image quality, it is critical to consider the task for which the image is being used. In the case of CT-based AC, there are two components to consider. The first is the calculation of the amount of attenuation that occurs for photons emitted from within the patient. To perform this calculation, the CT slice thickness is matched to that of the SPECT acquisition, typically 4 to $6 \mathrm{~mm}$, and the image is smoothed to match the SPECT spatial resolution. In addition, the attenuation is calculated as a sum along the photon's path of travel through the patient. All of this processing tends to average out the noise and allows accurate attenuation values to be obtained from what may appear to be very noisy CT images. The second component to consider is the registration of the CT to the SPECT study. Even with hybrid SPECT/CT cameras, the sequential acquisition of the CT and SPECT images increases the potential for patient movement between the images and thus the registration must always be assessed. Misregistration is recognized as a potential problem with SPECT-AC ${ }^{4}$ and can lead to artifacts. Even small shifts in registration, which may not lead to visually obvious errors, can still introduce small changes in the measured tracer uptake. Image registration is usually assessed visually in the clinic and subjectively corrected, when needed, by the camera operator. For this component of the task, noisier CT images may make it more difficult to evaluate the accuracy of the registration. Finally, to assess the quality of the AC, one should consider the task for which the corrected emission image will be used and determine what level of change is significant or clinically meaningful.

The paper presented in this issue of the Journal ${ }^{9}$ addresses CT dose reduction in the context of AC for half-dose stress-rest $(\sim 5 \mathrm{mSv})$ studies acquired on a dedicated cardiac camera. In 100 patient studies, the authors adjust the $\mathrm{x}$-ray tube voltage to acquire transmission maps at the standard $120 \mathrm{kVp}(0.58 \mathrm{mSv})$ as well as at 80 and $70 \mathrm{kVp}$ which reduces the patient dose to 0.19 and $0.12 \mathrm{mSv}$ respectively. They then assess the impact on the corrected images by measuring the change in relative uptake in each segment of 20-segment polar maps created from the attenuation-corrected SPECT images. They show that there is an excellent correlation between the images processed with the $70 \mathrm{kVp}$ and 80 $\mathrm{kVp}$ CT scans compared to the $120 \mathrm{kVp}$ scan (ICC coefficient $=0.99$ ) and that the bias is less than $1 \%$. Their Bland-Altman analysis showed a $95 \%$ confidence limit of $\pm 5 \%$ and that the results were similar for stress and rest.

To place these differences in context, the authors went on to do an intra- and inter-observer analysis of a subset of 20 patients. This analysis indicates the uncertainty in the clinical processing chain, that is, the variation in the measured perfusion that occurs simply by repeating the study. As the projection data are the same, ideally, the same tracer uptake would be measured when assessed a second time or by a second reader, but subjective assessments and manual interactions can lead to variations. With experienced readers, these variations are considered not to be clinically meaningful and thus provide an indication of the importance of any differences observed with different CT tube voltages.

This intra-/inter-reader analysis produced very similar results with a bias $<1 \%$, correlation of $\geq 0.97$ and Bland-Altman limits of $\pm 5 \%$. There was a suggestion of a small increase in the BA $95 \%$ confidence limits of $0.5 \%$ (or $5 \%$ relative increase in the total range), but an increase of this magnitude would not be clinically relevant. Given these results, the authors concluded that the dominant source of variation in the uptake values is user intervention in the processing chain that includes manual registration of the CT and emission data sets, re-orientation, and segmentation of the cardiac volume. The additional error introduced by lowering the voltage on the CT scan was minimal. The $120 \mathrm{kVp}$ CT could thus be exchanged for a $80 \mathrm{kVp}$ or even a $70 \mathrm{kVp} \mathrm{CT}$, with their corresponding reductions in patient dose, without loss in image quality.

An important limitation of the study is the size of the population considered. Attenuation and hence attenuation correction is strongly dependent on the size of the patient. The patients in this study had a mean BMI of $28 \mathrm{~kg} / \mathrm{m}^{2}$. To gain some insight into the weight-dependence of their results, the authors divided their group into tertiles and compared lowest (BMI $18-25 \mathrm{~kg} / \mathrm{m}^{2}$ ) to the highest (BMI $29-38 \mathrm{~kg} / \mathrm{m}^{2}$ ) tertile. They found very similar correlation, bias, and BA ranges for both and noted a slight increase in BA limits for the heavier group. However, the differences between processing with low- and standard-voltage CT scans remained dominated by the intra-/inter-observer variability. This 
suggests that the weight-dependence in the results is small over the range considered. Nevertheless, as patients increase in size, the risk of photon starvation increases which can lead to significant CT artifacts. The lower penetrating power of the reduced $\mathrm{kVp}$ x-ray beam means that starvation will occur in smaller patients at $70 \mathrm{kVp}$ than at $120 \mathrm{kVp}$, but the limits at which this introduces significant changes in uptake are unclear. Thus, further study is needed before implementing lower-voltage scans in a heavier patient population.

Dose reduction in MPI is a laudable goal, as long as it does not reduce image quality or the value of the test. The study by Grossman and colleagues provides important data to justify the use of lower-dose CT scans for AC in SPECT MPI.

\section{Disclosures}

The author has received research support from GE Healthcare.

\section{References}

1. National Council on Radiation Protection and Measurements. Ionizing radiation exposure of the population of the United States. Report no. 160. Bethesda, MD: National Council on Radiation Protection and Measurements; 2009.

2. Dorbala S, Blankstein R, Skali H, et al. Approaches to reducing radiation dose from radionuclide myocardial perfusion imaging. Nucl Med. 2015;56:592-9. https://doi.org/10.2967/jnumed.112. 115097.
3. Einstein AJ, Pascual TNB, Mercuri M, et al. Current worldwide nuclear cardiology practices and radiation exposure: Results from the 65 country IAEA Nuclear Cardiology Protocols Cross-Sectional Study (INCAPS). Eur Heart J. 2015;36:1689-96. https://doi.org/10. 1093/eurheartj/ehv117.

4. Dorbala S, Ananthasubramaniam K, Armstrong IS, et al. Single photon emission computed tomography (SPECT) myocardial perfusion imaging guidelines: Instrumentation, acquisition, processing, and interpretation. J Nucl Cardiol. 2018. https://doi.org/10. 1007/s12350-018-1283-y.

5. Van Dijk JD, Borren NM, Mouden M, et al. Effect of a patientspecific minimum activity in stress myocardial perfusion imaging using CZTSPECT: Prognostic value, radiation dose, and scan outcome. J Nucl Cardiol. 2018;25(1):26-35. https://doi.org/10.100 7/s12350-017-1011-z.

6. Einstein AJ, Johnson LL, DeLuca AJ, Kontak AC, Groves DW, Stant J, Pozniakoff T, Cheng B, Rabbani LE, Bokhari S. Radiation dose and prognosis of ultra-low-dose stress-first myocardial perfusion SPECT in patients with chest pain using a high-efficiency camera. J Nucl Med. 2015;56(4):545-51. https://doi.org/10.2967/ jnumed.114.150664 Epub 2015 Mar 5.

7. Siegel JA, Pennington CW, Sacks B. Subjecting radiologic imaging to the linear no-threshold hypothesis: A non sequitur of non-trivial proportion. J Nucl Med. 2017;58(1):1-6. https://doi.org/10.2967/ jnumed.116.180182.

8. Dorbala S, Blankstein R, Skali H, Park MA, Fantony J, Mauceri C, Semer J, Moore SC, Di Carli MF. Approaches to reducing radiation dose from radionuclide myocardial perfusion imaging. J Nucl Med. 2015;56(4):592-9. https://doi.org/10.2967/jnumed.112.115097.

9. Grossmann M, Giannopoulos AA, Bechtiger FA, Messerli M, Schwyzer M, Benz DC, Kudura K, Gebhard C, Gräni C, Pazhenkottil AP, Kaufmann PA, Buechel RR. Ultra-low-dose computed tomography for attenuation correction of cadmiumzinc-telluride single photon emission computed tomography myocardial perfusion imaging. J Nucl Cardiol. 2018. https://doi. org/10.1007/s12350-018-1303-y. 